\title{
BioLive - Location Based Biometric Attendance
}

\author{
Sheela Kathavate ${ }^{1}$, Shashank S. Kathavate ${ }^{2}$ \\ Associate Professor, Information Science and Engineering, BMS Institute of Technology and Management, Bangalore, \\ India $^{1}$ \\ Hexaware Technologies Ltd. Bangalore, India
}

\begin{abstract}
Having a lacklustre attendance system or process for employees creates inconsistency in their daily schedule. Employees coming to work a few minutes before the login time may face traffic at the attendance device causing incorrect data records to be registered although they were there well in advance. Organizations have to set up these devices all around the campus making it an expensive investment that may need further servicing throughout its use. Faulty devices will hinder employee login unless immediately fixed. This results in the need for an intelligent process to provide a simplified and flawless daily login for the employees. The absence of a technique capable of dealing with the abovestated complications led to the development of this innovation. This paper presents a novel and efficient technique integrating a smartphone application to simplify biometric login through the fingerprint scanner and a web application for information and a subscription-based package for users. A database stores the user information securely for verification purposes.
\end{abstract}

Keywords: Android Studio, Smartphone, Fingerprint Scanner, React Js.

\section{INTRODUCTION}

With the introduction of automation with the help of Artificial Intelligence, user's day to day activities have been simplified to great extents. Home automation devices, Internet of Things, robots, etc. have raised the expectations of people in the advancement of software technology. One of the latest advancements is location-based assistance with the help of sophisticated Global Positioning Systems (GPS) and tracking enabled applications, providing accurate and rapid data. It has helped in security, navigation, military applications, and many more. The location features enable novel ideas and can be used to assist attendance at organizations based on the smartphone location. This paper presents a dynamic and modern application to simplify daily biometric login for employees, henceforth called BioLive.

BioLive is an integrated application consisting of a smartphone application, web application, and database functionality each having distinct uses. The smartphone application provides the core functionality, enabling the entire login process and user interface features. The web application provides users with knowledge on the application along with a subscription-based plan one can purchase. The database server is responsible for storing and moving user data throughout the application; and does not store confidential information that may cause security threats such as the user's finger biometric. All confidential data is stored internally within the smartphone memory, making it known only to the user.

With the increase in the smartphones having a fingerprint scanner being shipped every year, nearly $70 \%$ of smartphones over two billion, it is evident that soon this will further raise, eventually being integrated into all smartphones. These fingerprint scanners are either optical or capacitive, optical scanners work based on the reflection of light on the skin and capacitive sensors make use of tiny capacitor array circuits that track the ridges in the user's fingerprint. This data is then converted into digital data with the help of an operational amplifier. Most smartphones use capacitive sensors which are extremely durable and accurate lasting for a long time. The scanned fingerprint is stored in the internal memory of the smartphone after encryption and can be accessed anytime by the device. This data is used by the smartphone application to perform user verification for the daily biometric login.

The smartphone application is the fundamental part of the system. It is responsible for biometric login and verification as well as providing attendance information for the user. The frontend and backend share data between each other to provide the user with an exceptional interface. The frontend takes relevant inputs and displays the output while the backend stores all the data in a relational database, sorting, filtering, and transferring the requested data to the frontend. The application makes use of the location features provided by open-source application programming interfaces (API) to simulate a device present on campus premises. Currently, the application is developed for Android based smartphones adaptable to other operating systems based on future needs.

The rest of the paper is organized as follows. In section 2 we explain relevant work. Section 3 highlights the proposed methodology. Section 4 highlights the smartphone application, web application and Database. Section 5 discusses the Results and section 6 concludes the paper. 


\section{International Journal of Advanced Research in Computer and Communication Engineering}

Vol. 10, Issue 1, January 2021

DOI 10.17148/IJARCCE.2021.10134

\section{RELATED WORK}

Uddin et al. [1] use location as a proof of attendance and proposed a new time and attendance system based on location. The application is installed on the user's phone. The application is associated with a unique user ID and location based on GPS. The monitoring software which receives and processes information from the mobile is installed on the workstation.

The authors Sultana et al. [2] proposed that the entry and leaving time of the employee can monitored by the GPS location tracker. This can be matched with the organization's GPS location and the attendance can be tracked.

Chiagozie et al. [3] have worked on a RFID system to produce time-attendance management. The RFID reader is connected to the host computer via a serial to USB converter cable. Visual basic.Net is used to develop the timeattendance system GUI. The time-attendance system provides the functionalities of the overall system such as displaying live ID tags transactions, registering ID, deleting ID, recording attendance.

RFID system has also been used to develop an attendance and monitoring system. RFID system basically consists of two components: the first one consists of tags and readers, and the second is a computer software or application. The authors Lim et al. [4] have proposed RFID based system to monitor attendance. The system is connected to the computer through RS232 or Universal Serial Bus (USB) port and stores the attendance taken in the database with real time clock capability of the system.

Mohamed et al. [5] proposed a fingerprint based attendance without the intervention of teacher. Students would be made to place their finger over the sensor so as to mark their presence in the class. It can communicate with a host computer using its USB interface. This device operates from a rechargeable battery. GUI application in host computer helps the teacher to manage the device and attendance.

A wireless fingerprint attendance management system has been proposed, designed and implemented by Yongqiang et al. [6]. This system based biometrics and wireless technique solves the problem of spurious attendance and the trouble of laying the corresponding network.

A wireless fingerprint attendance system based on ZigBee technology is proposed by Li et al. [7]. The system includes terminal fingerprint acquisition module and attendance management module in computer. It can realize automatically such functions as information acquisition of fingerprint, processing, wireless transmission, fingerprint matching, and attendance management.

Cayen et al. [8] proposed a wireless fingerprint attendance system which comprises at least one fingerprint scanner and a computer server. First templates of fingerprint related data are stored on the server, and similar templates are stored on the scanner. When a fingerprint is scanned, the fingerprint data is transmitted wirelessly to the server where a comparison is made. The server directs the scanner to display an indication whether or not a match was found. If available, an updated list of templates of fingerprint related data is transmitted from the server to the scanner.

A system in which fingerprint verification is done by using extraction of minutiae technique and the system that automates the whole process of taking attendance is proposed by Saraswat et al [9]. A fingerprint is captured by user interface which are likely to be an optical, solid state or an ultrasound sensor. The fingerprint verification is done by confirming the authenticity of one person by comparing his/her captured fingerprint templates against the stored templates in database.

Kumbhar et al. [10] proposed the development of Attendance Monitoring System (AMS) using Android platform. The basic requirement of the AMS is an android device which will run the application with the help of which the student's will mark their attendance. The other requirement is a personal computer on the server side which will store the database.

Pankanti et al. [11] derive an expression which estimates the probability of falsely associating minutiae-based representations from two arbitrary fingerprints. For example, the probability that a fingerprint with 36 minutiae points will share 12 minutiae points with another arbitrarily chosen fingerprint with 36 minutiae points is $6.10 \times 10-8$. These probability estimates are compared with typical fingerprint matcher accuracy results.

An Automated Fingerprint Attendance System (AFAS) which is a highly specialized system that records students' attendance by comparing a single fingerprint image with the fingerprint images previously stored in a database was proposed by Shoewu et al. [12]. The fingerprint is unique to each individual and cannot be shared. It allows students to register for lectures with ease and eliminate errors that are associated with attendance reports because the system generates reports at the end of the semester.

Our proposed system uses the combined technique of using the fingerprint with the android mobile phone and integrating it with the GPS location.

\section{Proposed SySTEM}

BioLive aims to improve the daily login process used by organizations for their employees by providing a modern novel application. The application replaces the traditional biometric scanners present around the establishment. Utilizing the location functions provided by the smartphone, the exact perimeter of the campus can be contemplated on the map. The 


\section{International Journal of Advanced Research in Computer and Communication Engineering}

Vol. 10, Issue 1, January 2021

DOI 10.17148/IJARCCE.2021.10134

user on entering the perimeter is allowed to immediately login for their daily attendance, hassle-free. The same device can be used by multiple users, however, each of them has to be within the perimeter during the biometric scan through the smartphone fingerprint scanner. This reaches all users in case of damages to their scanner, misplaced smartphone, or incompatibility. Once logged, BioLive provides the user with details of the login time and expected logout time. Once ready to logout for the day, the user simply needs to select the logout button and scan their fingerprint again. The application shows the users their login and logout time for the past week and month based on their preference. The webpage has a home page guiding the user and giving an overall idea about BioLive. The webpage also is responsible for signing up new organizations, where the administrator can select their preferred plan based on the number of employees. The website also collects feedback and provides constant information on the latest updates and offers. Fig. 1 shows the block diagram of BioLive and the overall flow from the launch of the application till shutdown.

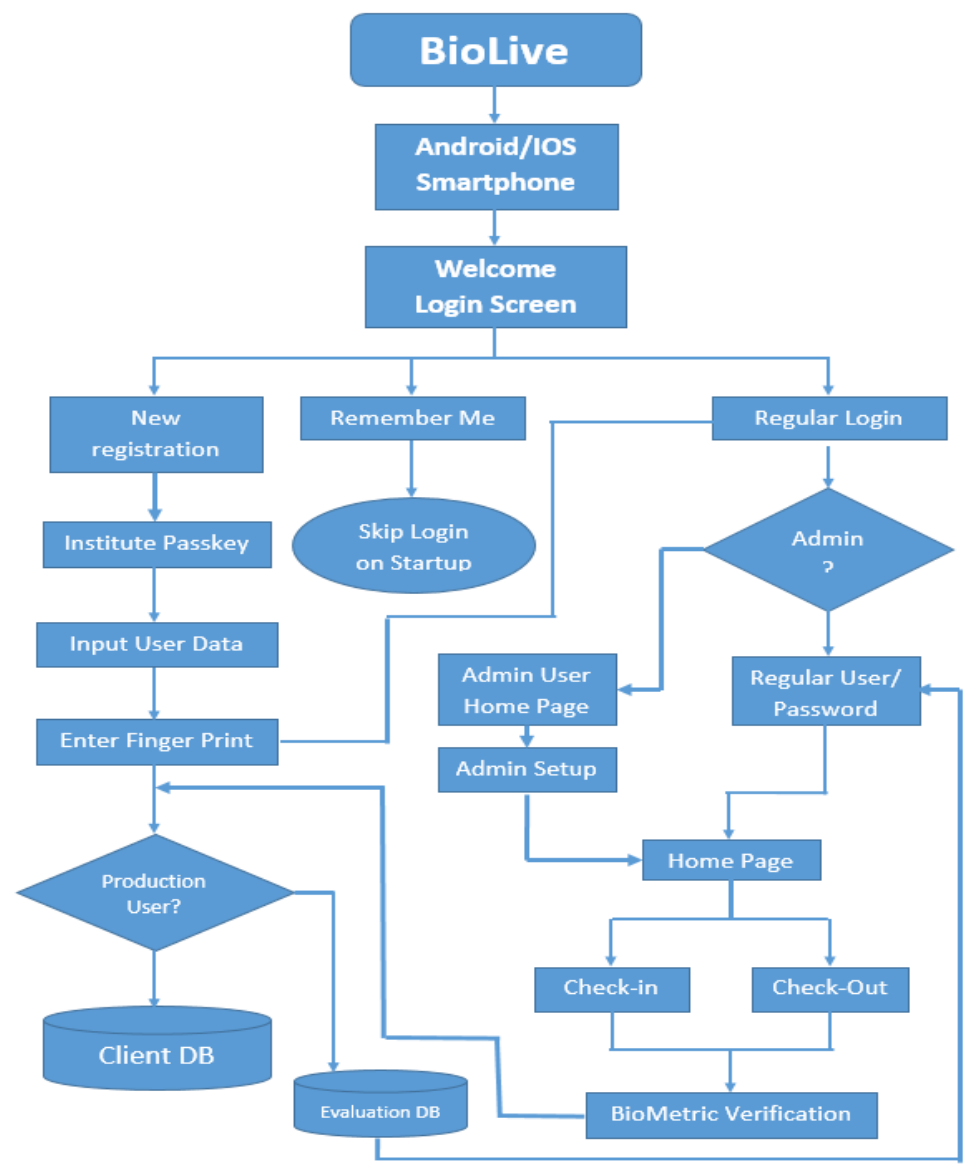

Fig. 1 Biolive block diagram

\section{IMPLEMENTATION}

\section{A. Smartphone Application}

The smartphone application is responsible for the core functionality of BioLive. The application provides the user with many functions to provide smooth registration and usage at all times. The application consists of a login screen, home screen, biometric login screen, log history screen, profile screen and a settings screen. Each organization is given an administrator who is automatically detected based on the login credentials and who has special privileges namely, setting the perimeter, adding users and removing users.

\section{1) Login/Sign Up screen:}

This is the first page of the application which requests the user to login to their BioLive accounts as in Fig 2 . A user who has been added by the administrator may create a new account on the same page using the signup button with a special one-time key. In case the user forgets their password a 'forgot password' button is provided for the user to reset their password using email or mobile verification. After successfully creating an account and logging in, the user may tick the 'remember me' checkbox to skip the login screen on further launches going directly to the home screen. 


\section{DOI 10.17148/IJARCCE.2021.10134}

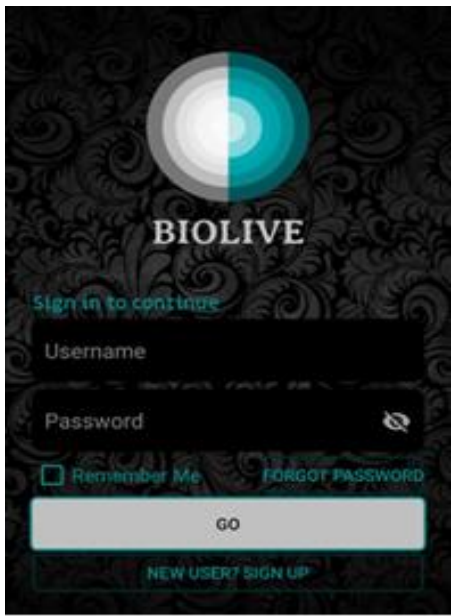

Fig. 2 Login Screen

Home screen:

The home screen provides the user with a map zoomed in on their location at launch and later shifts to the organization's location filling the perimeter green as in Fig 3 if the user is present within, otherwise filling it red as in Fig 4. The map refreshes every few seconds showing the user's new location with respect to the organization's perimeter. On successfully entering the perimeter the user may now select the 'check in' or 'check out' button to login or logout with

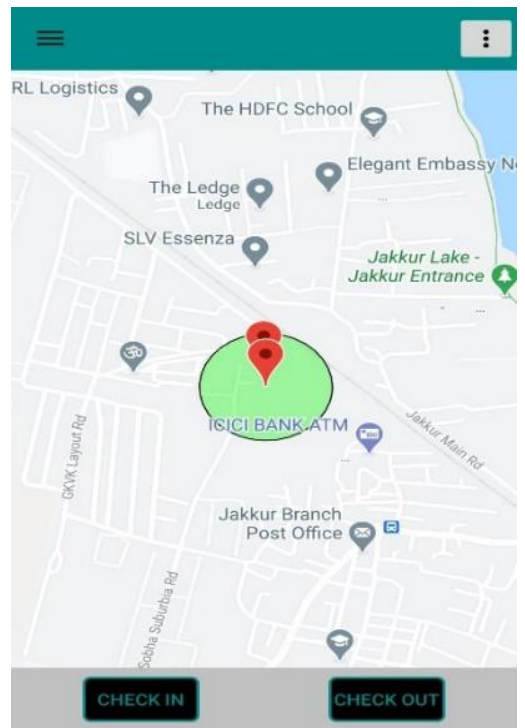

Fig. 3 Inside the perimeter

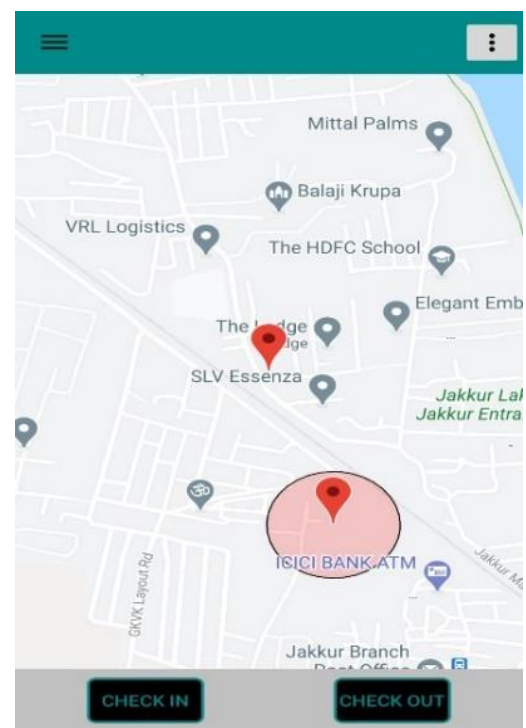

Fig. 4 Outside the perimeter

the fingerprint scanner. The screen also provides the user with a navigation menu as in Fig 5 containing buttons to show the user's log history, profile and friends. The user may log in from their account using the logout button provided in the navigation menu. The user may also select a drop-down, providing settings and information about BioLive. The admin can use special buttons provided in this drop-down not visible to normal users. 


\section{International Journal of Advanced Research in Computer and Communication Engineering}

Vol. 10, Issue 1, January 2021

DOI 10.17148/IJARCCE.2021.10134

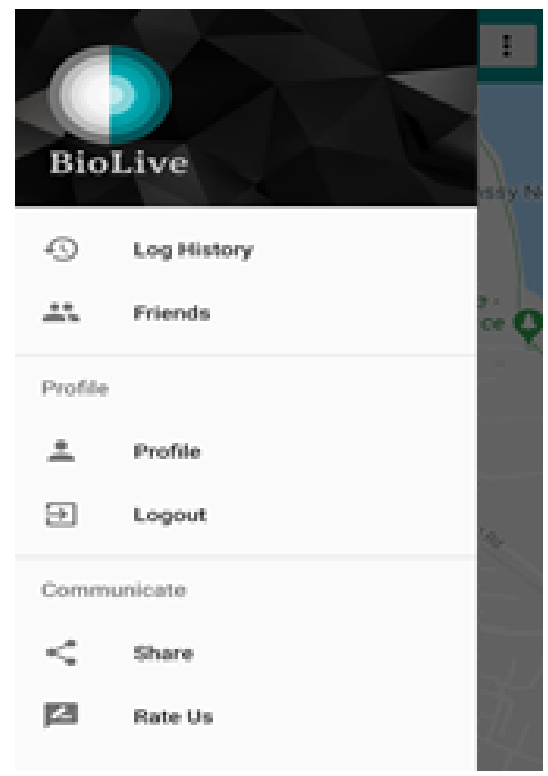

Fig. 5 Home screen navigation

Admin Screens:

The admin is provided with three additional options, setting the perimeter, adding users and removing users as in Fig 6. The perimeter is set using adjacent map pins as the vertices later joined to form a closed polygon and a higher accuracy is achieved with more number of pins as in Fig 7. The admin may add more users by going through his phone contacts and inviting the user with a key and a message. The admin may also remove users present in the database by deleting the users selected. Multiple users can be added and removed at the same time selecting multiple checkboxes.

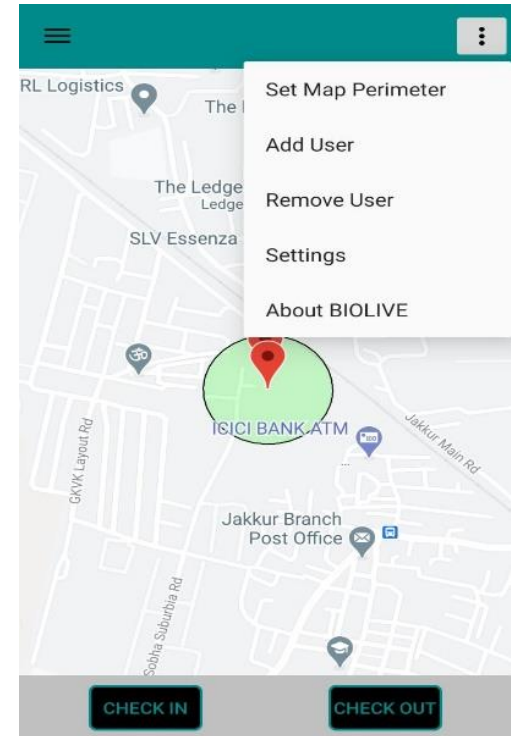

Fig. 6 Admin specific options

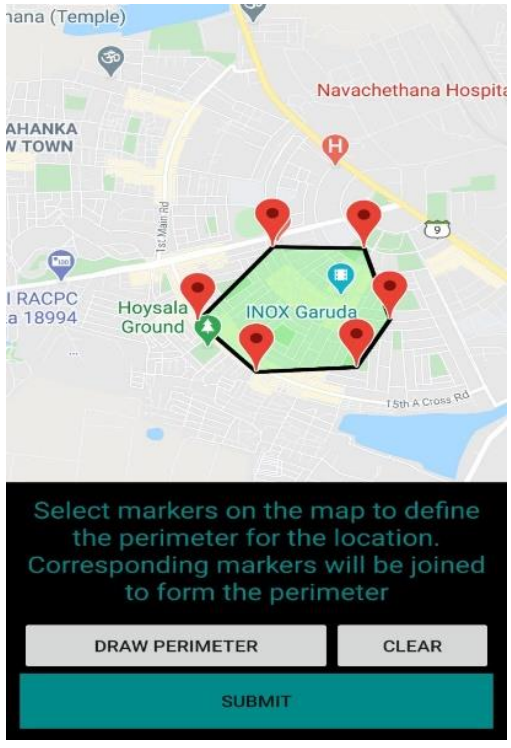

Fig. 7 Perimeter setting

Once the user selects the 'check in' or 'check out' button he is guided to the fingerprint screen as in Fig 8 where the smartphone's fingerprint scanner is activated requesting them to physically place their finger on it. On successful verification, a message is shown and the user's login or logout time is stored in the database. In case of failure in verification as in Fig 9, the user has up to 5 attempts after which he/she must wait one min to try again. 


\section{International Journal of Advanced Research in Computer and Communication Engineering}

Vol. 10, Issue 1, January 2021

DOI 10.17148/IJARCCE.2021.10134

The application is programmed using Java and Extensible Markup Language (XML) in Android Studio, Google's opensource Android application development software. It is an extremely powerful Integrated Development Environment (IDE) built on JetBrains' IntelliJ IDEA software specifically designed for android development.

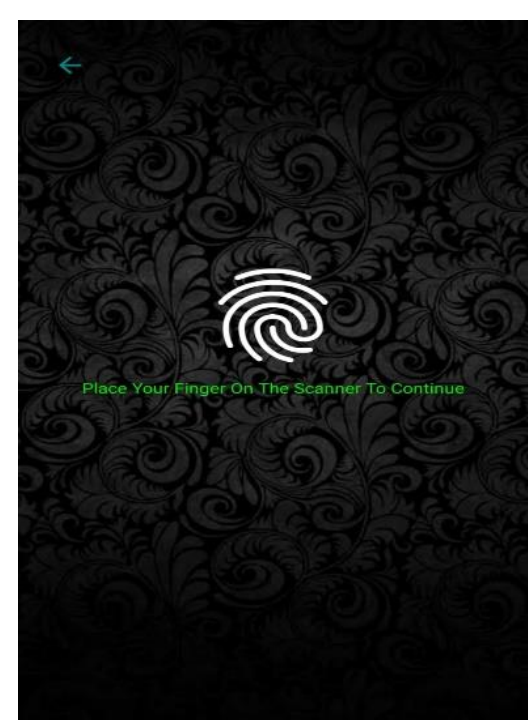

Fig. 8 Fingerprint screen

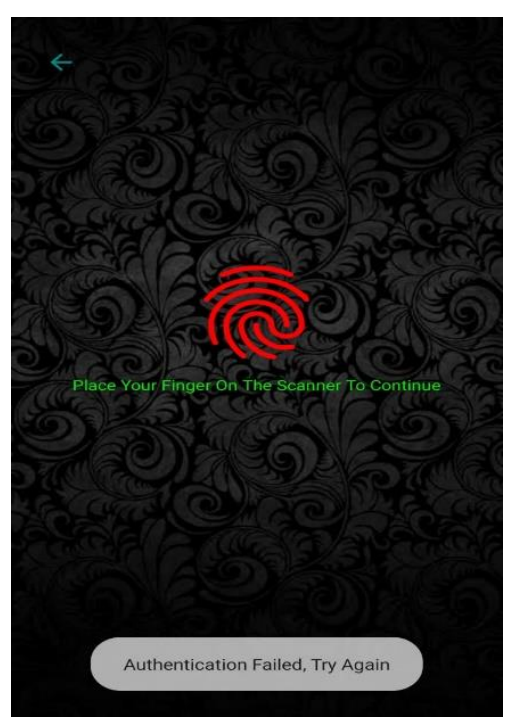

Fig. 9 Authentication Failed

5)

\section{Settings screen:}

The user may modify various settings of the application ranging from user profile credentials to user interface settings as shown in Fig 10. The settings page can be accessed from the drop down menu on the top right of the screen.

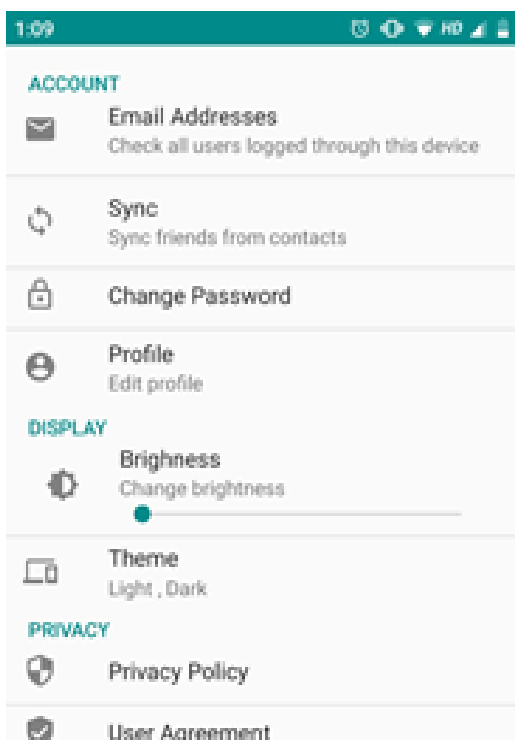

Fig. 10 Profile Settings

\section{B. Web Application}

The application website is responsible for two major processes, providing information about BioLive and providing subscription-based packages for organizations to purchase. The webpage also has user feedback and contact information for and issues faced.

\section{1) Home page:}

The home page contains information on new releases and offers. The page also contains navigation buttons to the products page, about page, Login page and connect-with-us page as in Fig. 11. 


\section{International Journal of Advanced Research in Computer and Communication Engineering}

Vol. 10, Issue 1, January 2021

DOI 10.17148/IJARCCE.2021.10134

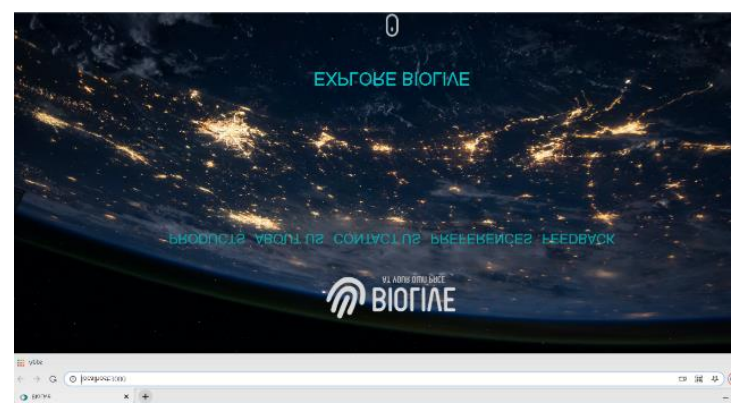

Fig. 11 Home Page

Product page:

The product page as in fig. 12 provides the organization with the available plans. The organization may sign up for a free month trial and further follow a subscription plan based on the months required. Different plans are available based on the number of employees in the organization making it flexible. On providing all the required information the admin receives their user login credentials which can be used to log in to the smartphone application.

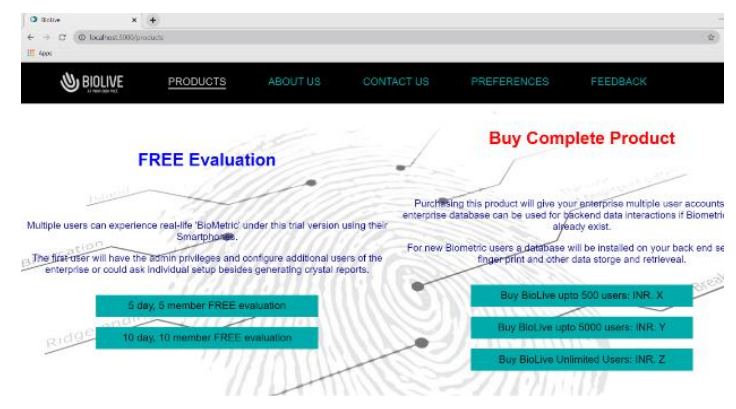

Fig. 12 Product page

\section{Database}

The database is responsible for storing and sharing the data with both the smartphone application and web application. The database being used is MySQL, an open-source relational database. Various tables of data pertaining to the respective section are linked together based on a parameter refining data access and retrieval. The application runs multiple queries internally during operation to obtain the necessary information from the database. This database is present on a local server which can be accessed over the internet by the users. The database stores information such as user name, password, date of birth, email ID, organization, sex, phone number and address. When the user logs into the application the data application verifies if the entered data is the same as present in the database. If true then the user may proceed further, otherwise, the user must follow the specified steps.

\section{RESUlts}

The application was tested by 20 different user login credentials. The first few were tested when the user was well within the perimeter and this test was successful and the user could log in through the fingerprint scanner. The next few tests were performed when the user was outside the perimeter and this test didn't allow the user to login with the fingerprint scanner. A few tests were performed right on the perimeter and the user was allowed to login proving to be extremely accurate. A few attempts were performed by other users in different user accounts which was unsuccessful.

\section{Conclusion}

BioLive fulfils its purpose by providing a novel working procedure to enhance user experience with the daily biometric login. It helps ensure perfect timing for all employees, by virtually creating multiple biometric scanners. Prior processes used for daily biometric, involved individual devices kept in the building can be cumbersome and can cause delays in employee login times. This paper proposes a novel method integrating a smartphone application, web application and database connectivity to enable the remote login functionality. With the rapid increase in manufacturing fingerprint scanner enabled smartphones, this idea is easily implementable everywhere. 


\section{International Journal of Advanced Research in Computer and Communication Engineering}

Vol. 10, Issue 1, January 2021

DOI 10.17148/IJARCCE.2021.10134

\section{REFERENCES}

[1]. Uddin, Mohammad Salah, S. M. Allayear, N. C. Das, and F. A. Talukder. "A location based time and attendance system." International journal of computer theory and engineering 6, no. 1 (2014): 36

[2]. Sultana, Shermin, Asma Enayet, and Ishrat Jahan Mouri. "A smart location based time and attendance tracking system using android application." International Journal of Computer Science, Engineering and Information Technology (IJCSEIT) 5, no. 1 (2015): 1-5.

[3]. Chiagozie, Ononiwu G., and Okorafor G. Nwaji. "Radio frequency identification (RFID) based attendance system w/ automatic door unit" Academic Research International 2, no. 2 (2012): 168.

[4]. Lim, T. S., S. C. Sim, and M. M. Mansor. "RFID based attendance system." In 2009 IEEE Symposium on Industrial Electronics \& Applications, vol. 2, pp. 778-782. IEEE, 2009

[5]. Mohamed, Basheer KP, and C. V. Raghu. "Fingerprint attendance system for classroom needs." In 2012 Annual IEEE India Conference (INDICON), pp. 433-438. IEEE, 2012.

[6]. Yongqiang, Zhang, and Liu Ji. "The design of wireless fingerprint attendance system." In 2006 International Conference on Communication Technology, pp. 1-4. IEEE, 2006.

[7]. Li, Jian-po, Xu-ning Zhu, Xue Li, Zhi-ming Zhang, and Ji-sheng Sui. "Wireless fingerprint attendance system based on zigbee technology." In 2010 2nd International Workshop on Intelligent Systems and Applications, pp. 1-4. IEEE, 2010.

[8]. Cayen, Joseph D. "Wireless fingerprint attendance system." U.S. Patent 7,826,645, issued November 2, 2010.

[9]. Saraswat, Chitresh, and Amit Kumar. "An efficient automatic attendance system using fingerprint verification technique." International Journal on Computer Science and Engineering 2, no. 02 (2010): 264-269.

[10]. Kumbhar, A. A., Wanjara, K. S., Trivedi, D. H., Khairatkar, A. U., \& Sharma, D. (2014). “Automated Attendance Monitoring System using Android Platform.”, International Journal of Current Engg and Technology, Vol. 4, No. 2, pp1096-1099.

[11]. Pankanti, S., Prabhakar, S., \& Jain, A. K. (2002). “On the individuality of fingerprints”, Pattern Analysis and Machine Intelligence, IEEE Transactions on, 24(8), 1010-1025.

[12]. Shoewu, O. and O.A. Idowu (2012), "Development of Attendance Management System using Biometrics", Pacific Journal of Science and Technology, 13(1):300-307. 